\title{
The Einstein-Yang-Mills equations from Bianchi identities
}

\author{
Christian G. Böhmeı* \\ Department of Mathematics, University College London, Gower Street, London, WC1E 6BT, UK and \\ Institute of Cosmology $\&$ Gravitation, University of Portsmouth, Portsmouth PO1 2EG, UK \\ Luca Fabbri甲 \\ Theory Group, INFN - Department of Physics, University of Bologna, Via Irnerio 46, C.A.P. 40126, Bologna, Italy
}

(Dated: July 21, 2021)

\begin{abstract}
We suggest a novel extension to the Kaluza-Klein scheme that allows us to obtain consistently all $S U(n)$ Einstein-Yang-Mills theories. This construction is based on allowing the five-dimensional spacetime to carry some non-vanishing torsion; however, the four-dimensional spacetime remains intrinsically torsion-free.
\end{abstract}

PACS numbers: $04.50 .+\mathrm{h}, 11.25 . \mathrm{Mj}$

\section{INTRODUCTION}

The great success of Einstein gravity opens deeper philosophical issues: If we assume that any physical theory should be generally covariant, then it is difficult to understand why all the other interactions are not described side by side with gravity in the framework of General Relativity.

Kaluza [1] and Klein [2] proposed to pursue the electrogravitational unification by enlarging the spacetime to a 5-dimensional spacetime, in which the extra dimension was thought to carry the information concerning electromagnetism (for general reviews, see e.g. [3, 44]). By compactification of this fifth dimension, the resulting theory could describe properly the Einstein-Maxwell theory; for some critical account see [5]. Such compactifications became of great interest in the context of higher dimensional theories. These higher dimensional spacetimes are important due to the fact that they can host supergravity or (super) string theories.

On the other hand, Weyl [6] in 1918 tried to extend General Relativity by considering a conformally rescaled metric $g_{a b} \rightarrow \phi g_{a b}$. The covariant derivative of the rescaled metric does not necessarily vanish and leads to non-metricity which was considered to be related the electromagnetic potential. Weyl, as many others later, attempted to include electromagnetism in a theory already containing gravity, hoping to accomplish their formal unification. Details on the history of such unified field theories can be found in [7]. Although Weyl's original idea had many problems of its own, it later went on to become the key ingredient for gauge field theories, the building blocks of the Standard Model of particle physics.

Once tensor calculus was recognized as the geometrical structure for General Relativity, the symmetry of the connection was established in order to represent the Principle of Equivalence in a 4-dimensional spacetime. On

\footnotetext{
*Electronic address: c.boehmer@ucl.ac.uk
}

${ }^{\dagger}$ Electronic address: Luca.Fabbri@bo.infn.it this background, the simplest differential identities of the theory, namely the Bianchi identities, implied the existence of conservation laws. This match between geometrical (Einstein tensor) and physical (energy-momentum tensor) quantities was the key point to satisfy Mach's principle on the origin of masses, which motivated the form of the gravitational field equations.

Ever since Weyl's approach, similar unification ideas were proposed. During the 1920s, Cartan, see e.g. [8] and later works, tried to find the room necessary to incorporate electromagnetism into General Relativity by removing the assumption on the symmetry of the connection, thereby introducing the concept of torsion [9, 10]. In these torsion theories the connection contains a skewsymmetric part, the Cartan or torsion tensor, which plays the role of a physical quantity. Often the torsion of the spacetime is related to the spin of the particle, so that mass couples to the curvature of spacetime whereas spin couples to its torsion.

There are also some recent attempts to understand the neutrino in the context of geometries with torsion. The Weyl Lagrangian turns out to be equivalent to a purely geometrical Lagrangian [1, 12].

Yet another way is possible. One can take into account both the possibility of a higher dimensional spacetime and the possibility that the corresponding higher dimensional torsion is non-zero [13, 14, 15, 16]. The geometrical approach used by Einstein can easily be extended in order to consider higher dimensional manifolds and the corresponding Bianchi identities. These will be used to define higher dimensional conservation laws.

For a thorough analysis of the Kaluza-Klein scheme in the presence of torsion and gauge fields, we refer the reader to Kalinowski [13] and references therein. In general, for higher order gauge fields, a $(4+\operatorname{dim}(\mathscr{G}))$ dimensional manifold has been considered in the past.

Another interesting approach in that direction is the 5-dimensional projective unified theory of Schmutzer 17, 18] where the effective 4-dimensional theory is obtained by using a postulated projection tensor to reduce the higher-dimensional theory. However, it has been shown that this theory is in disagreement with experiments un- 
less modified by assuming a totally antisymmetric torsion tensor [19]. Note that we will consider the 5-dimensional Bianchi identities in order to derive the effective theory.

So far, however, all approaches to extend the original Kaluza-Klein scheme in order to also describe higher order gauge fields with semi-simple and compact Lie group $\mathscr{G}=S U(n)$, have not yielded promising results. The main reason for this is that the gauge potentials and field strengths have an internal group index. This makes it difficult to add a geometrical quantity linear in the potential, as in the $U(1)$ case.

In the present work, we will consider a framework in which the higher dimensional torsion is non-vanishing but the 4-dimensional spacetime is torsion-free. Since we are not considering fermionic matter sources such an assumption is well motivated. Moreover we will introduce a set of auxiliary vector fields $u^{\mathfrak{a}}$, which span a real $\operatorname{dim}(\mathscr{G})$ dimensional vector space $\mathbb{R}^{\operatorname{dim}(\mathscr{G})}$. We show that this approach will enable us to obtain the complete $S U(n)$ Einstein-Yang-Mills equations.

\section{KALUZA-KLEIN REDUCTION WITH TORSION}

Let us recall the relevant geometrical equations necessary to compactify a 5 -dimensional spacetime to a 4dimensional one. Assume that the $5 \mathrm{~d}$ manifold is allowed to be torsioned, however, the $4 \mathrm{~d}$ manifold is assumed to be torsion-free, in agreement with all observations so far. Let the metric be of the following form

$$
\begin{array}{r}
d s^{2}=\eta_{m n} e^{m} \otimes e^{n}=\eta_{a b} e^{a} \otimes e^{b}-e^{5} \otimes e^{5}, \\
e^{5}=d x^{z}, \quad d e^{5}=0,
\end{array}
$$

where the indices of the middle of the alphabet $(m, n, \ldots)$ are complete spacetime indices and letters from the beginning of the alphabet $(a, b, \ldots)$ take values $1,2,3,4$ and letters from the end $(z)$ take the value 5 . We choose $\eta_{m n}=\operatorname{diag}(+1,-1,-1,-1,-1)$, see [20, 21].

The following relations define the torsion 2 -form to be

$$
\tilde{T}^{m}=D e^{m}=d e^{m}+\omega^{m}{ }_{n} \wedge e^{n} .
$$

In order to further simplify the torsion contributions due to the presence of the $5 \mathrm{~d}$ torsion, we moreover assume $\omega^{z}{ }_{a z}=0$ (the $z$-component of the connection 1-form $\left.\omega^{z}{ }_{a}=0\right)$. According to our metric, we may split the index $m=(a, z)$ which yields

$$
\begin{aligned}
& \tilde{T}^{a}=D e^{a}=d e^{a}+\omega^{a}{ }_{b} \wedge e^{b}+\omega^{a}{ }_{z} \wedge e^{z}, \\
& \tilde{T}^{z}=D e^{z}=\omega^{z}{ }_{a} \wedge e^{a} .
\end{aligned}
$$

The first torsion 2-form $\tilde{T}^{a}$ can further be split into an intrinsically $4 \mathrm{~d}$ torsion part $T^{a}$ and another part coming from the additional spacetime structure of the fifth dimension. Since we assume the $4 \mathrm{~d}$ spacetime to be torsion free, we take the intrinsic torsion part to vanish, $T^{a}=0$.
This simply states that the $4 \mathrm{~d}$ spin-connection can be computed from the vielbein $e^{a}, \omega^{a}{ }_{b}=\omega^{a}{ }_{b}(e)$, whereas the spin-connection components $\omega^{a}{ }_{z}$ yield an additional field contribution to the $4 \mathrm{~d}$ spacetime

$$
\begin{aligned}
& \tilde{T}^{a}=\omega^{a}{ }_{z} \wedge e^{z}, \\
& \tilde{T}^{z}=\omega^{z}{ }_{b} \wedge e^{b} .
\end{aligned}
$$

We expand $\omega^{z}{ }_{b}$ in terms of basis vielbeins and write Eq. (7) to arrive at

$$
\tilde{T}^{z}=\omega_{b a}^{z} e^{a} \wedge e^{b}=F_{a b} e^{a} \wedge e^{b}
$$

where we introduced the 2 -form $F$. The components of $F$ are those of the spin-connection component of the additional dimension $F_{a b}=\omega^{z} b a$. Therefore, equation (8) is simply

$$
\tilde{T}^{z}=F_{a b} e^{a} \wedge e^{b}=F .
$$

From the definition of $F_{a b}$ we can now easily find the analog expression involving $\omega^{b} z$

$$
F_{a b}=\omega_{b a}^{z}=-\omega_{b}^{z}{ }_{a}=\omega_{b z a}
$$

which after raising the index $b$ simply yields

$$
\omega_{z a}^{b}=F_{a}^{b}, \quad \omega_{z}^{b}=F_{a}^{b} e^{a} .
$$

Hence, from (6) the torsion 2-form $\tilde{T}^{a}$ becomes

$$
\tilde{T}^{a}=F_{b}{ }^{a} e^{b} \wedge e^{z}
$$

The curvature 2 -form is defined by

$$
\tilde{R}_{n}^{m}=\left(D^{2}\right)^{m}{ }_{n}=d \omega^{m}{ }_{n}+\omega^{m}{ }_{l} \wedge \omega^{l}{ }_{n}
$$

which according to the metric splits as follows

$$
\begin{aligned}
\tilde{R}_{b}^{a} & =d \omega^{a}{ }_{b}+\omega^{a}{ }_{c} \wedge \omega_{b}^{c}+\omega^{a}{ }_{z} \wedge \omega^{z}{ }_{b}, \\
\tilde{R}^{a}{ }_{z} & =d \omega^{a}{ }_{z}+\omega^{a}{ }_{b} \wedge \omega^{b}{ }_{z}, \\
\tilde{R}^{z}{ }_{a} & =d \omega^{z}{ }_{a}+\omega^{z}{ }_{b} \wedge \omega^{b}{ }_{a},
\end{aligned}
$$

The last term of $\tilde{R}_{b}^{a}$ can be re-written in terms of $F$ and becomes

$$
\omega^{a}{ }_{z} \wedge \omega_{b}^{z}=F_{c}{ }^{a} F_{d b} e^{c} \wedge e^{d}
$$

Since the first two terms of $\tilde{R}_{b}^{a}$ are the intrinsic 4 d curvature of that spacetime we find

$$
\tilde{R}_{b}^{a}=R_{b}^{a}+F^{a}{ }_{c} F_{b d} e^{c} \wedge e^{d}
$$

For the other curvature 2-form components we get

$$
\begin{aligned}
\tilde{R}^{a}{ }_{z} & =D F_{c}{ }^{a} \wedge e^{c}, \\
\tilde{R}^{z}{ }_{a} & =D F_{c a} \wedge e^{c},
\end{aligned}
$$

where we took into account $\tilde{R}^{a}{ }_{z}=-\tilde{R}_{z}{ }^{a}=\tilde{R}^{z a}$. From these one can compute the Ricci 1 -forms and the Ricci 
scalar and the resulting Einstein 1-form which yields the following (anholonomic) components of the Einstein tensor

$$
\begin{aligned}
\tilde{G}_{a b} & =G_{a b}-F_{a c} F_{b}^{c}-\frac{1}{2} F_{c d} F^{c d} \eta_{a b}, \\
\tilde{G}_{z b} & =\nabla_{a} F_{b}{ }^{a}, \\
\tilde{G}_{z z} & =\frac{1}{2} R+\frac{1}{2} F_{c d} F^{c d} .
\end{aligned}
$$

\section{BIANCHI IDENTITIES AND FIELD EQUATIONS}

The contracted third Bianchi identity $\left(D \tilde{R}_{b}^{a}=0\right)$ yields the vanishing of the covariant derivative of the Einstein tensor, which is one of the most important aspects of the gravitational field equations. The conservation of energy-momentum can be regarded solely as a consequence of the geometry of spacetime.

In our $4+1$ approach, the contracted Bianchi identity splits into two pieces

$$
\begin{aligned}
\nabla^{b} G_{a b} & =F_{a c}\left(\nabla^{b} F_{b}^{c}\right), \\
\nabla^{b}\left(\nabla_{a} F_{b}{ }^{a}\right) & =0 .
\end{aligned}
$$

These equations can be re-written in a more familiar form. Since we assume that the $4 \mathrm{~d}$ spacetime is intrinsically torsion-free and moreover that $F_{a z}=0$, the following (purely) geometrical relation is valid

$$
\nabla_{a} F_{b c}+\nabla_{b} F_{c a}+\nabla_{c} F_{a b}=0
$$

which implies that

$$
\frac{1}{4} \nabla_{c}\left(F_{a b} F^{a b}\right)=-F^{a b} \nabla_{a} F_{b c} .
$$

If we now use equations (25) and (27) to rewrite the right-hand side of (24), the following is found

$$
\nabla^{b} G_{a b}=\nabla^{b}\left(F_{a c} F_{b}^{c}+\frac{1}{4} \eta_{a b} F_{c d} F^{c d}\right) .
$$

This immediately implies the identification of the righthand side with the energy-momentum tensor of the electromagnetic field

$$
T_{a b}=F_{a c} F_{b}^{c}+\frac{1}{4} \eta_{a b} F_{c d} F^{c d}, \quad U(1) \text { gauge theory. }
$$

Before proceeding further and generalizing the above the arbitrary $S U(n)$ gauge groups, it is important to show the consistency of our above result with the recent criticism put forward by Hehl [22, 23, 24]. Hehl argued that the electromagnetic field strength cannot be simply related to the torsion of spacetime in a $4 \mathrm{~d}$ spacetime. The main geometrical argument behind that is based on the fact that the torsion 1-form decomposes into three irreducible pieces and that one must relate the field strength to any of these pieces rather than some suitable combination. In general, the components of the torsion tensor decompose into irreducible components (a vector part, an axial vector part and a tensor part) as

$$
\begin{aligned}
T^{m} & ={ }^{(1)} T^{m}+{ }^{(2)} T^{m}+{ }^{(3)} T^{m}, \\
\frac{1}{2} n^{2}(n-1) & =\frac{1}{3} n\left(n^{2}-4\right)+n+\frac{1}{6} n(n-1)(n-2),
\end{aligned}
$$

which in four dimensions becomes

$$
24=16+4+4 .
$$

Since no irreducible piece has 6 independent components, none can directly account for the Abelian field strength. In our approach, however, we are considering a $5 \mathrm{~d}$ space, for which the decomposition reads

$$
50=35+5+10 .
$$

If we furthermore take into account that we assumed the intrinsically $4 \mathrm{~d}$ spacetime to be torsion-free, then the resulting $5 \mathrm{~d}$ torsion tensor has less allowed components, namely the difference of (33) and (32), and we find the following number of allowed irreducible components

$$
\begin{aligned}
(50-24) & =(35-16)+(5-4)+(10-4), \\
26 & =19+1+6 .
\end{aligned}
$$

Therefore, in the torsioned Kaluza-Klein scheme, there exists one (and only one) irreducible component of the higher dimensional torsion tensor that has 6 independent components, the axial torsion vector part, if the intrinsic $4 \mathrm{~d}$ torsion is assumed to be zero. It is worth noting that it has been argued recently [25], that the torsion tensor should be totally antisymmetric, quite independent of the model, by assuming a strong metricity argument. This observation, as well as our result, are also in agreement with the suggested modifications [19] in the context of projective unified theory [17, 18].

So far $F_{a b}$ may only account for the Abelian $U(1)$ field strength. However, it is our aim to find the EinsteinYang-Mills equations for any compact, semi-simple gauge group $\mathscr{G}$. The Lie algebra of $\mathscr{G}$ is characterized by the commutation relations $\left[T_{\mathfrak{a}}, T_{\mathfrak{b}}\right]=i f_{\mathfrak{a} \mathfrak{b}}{ }^{\mathfrak{c}} T_{\mathfrak{c}}, \mathfrak{a}, \mathfrak{b}, \mathfrak{c}=$ $1, \ldots, \operatorname{dim}(\mathscr{G})$, where $T_{\mathfrak{a}}$ are the generators of the Lie algebra. Furthermore we have the relations $\operatorname{tr}\left(T_{\mathfrak{a}}\right)=0$, for all $\mathfrak{a}$ and $\operatorname{tr}\left(T_{\mathfrak{a}} T_{\mathfrak{b}}\right)=K \delta_{\mathfrak{a} \mathfrak{b}}$, where $K>0$ is a normalization factor.

We have up to now assumed that the geometrical quantities are not Lie algebra valued and therefore we cannot simply replace $F_{a b}$ by $F_{a b}^{\mathfrak{a}} T_{\mathfrak{a}}$. Neither can we use the trace $\operatorname{tr}\left(F_{a b}^{\mathfrak{a}} T_{\mathfrak{a}}\right)$, since $\operatorname{tr}\left(T_{\mathfrak{a}}\right)=0$ and therefore this term would also vanish. On the other hand, due to the contractions to get the Einstein tensor, the torsion and hence the terms with $F_{a b}$ would appear quadratically, as expected from the known form of the Yang-Mills energy-momentum tensor. Hence, we cannot use a term 
quadratic in the field strength, as this would yield a quartic term. However, these problems can be circumvented by introducing an auxiliary field $U^{\mathfrak{a}}$ in the following way

$$
F_{a b} \rightarrow \operatorname{tr}\left(F_{a b}^{\mathfrak{a}} T_{\mathfrak{a}} U^{\mathfrak{b}} T_{\mathfrak{b}}\right)=K F_{a b}^{\mathfrak{a}} U^{\mathfrak{b}} \delta_{\mathfrak{a} \mathfrak{b}} .
$$

For the Abelian group $U(1)$ there is only one generator and no auxiliary field is needed.

Let us now apply the substitution (35) to the above energy-momentum tensor (29)

$$
\begin{aligned}
T_{a b}= & \operatorname{tr}\left(F_{a c}^{\mathfrak{a}} T_{\mathfrak{a}} U^{\mathfrak{b}} T_{\mathfrak{b}}\right) \operatorname{tr}\left(F_{b}^{c}{ }^{\mathfrak{a}} T_{\mathfrak{a}} U^{\mathfrak{b}} T_{\mathfrak{b}}\right) \\
& +\frac{1}{4} \eta_{a b} \operatorname{tr}\left(F_{c d}^{\mathfrak{a}} T_{\mathfrak{a}} U^{\mathfrak{b}} T_{\mathfrak{b}}\right) \operatorname{tr}\left(F^{c d \mathfrak{a}} T_{\mathfrak{a}} U^{\mathfrak{b}} T_{\mathfrak{b}}\right) .
\end{aligned}
$$

In the following we will analyze the products of the two trace terms. For further simplification we choose $\mathscr{G}=S U(2)$, but the principal result applies to any gauge group $\mathscr{G}=S U(n)$; further note that the Lie algebra of $\mathscr{G}$ has $\operatorname{dim}(\mathscr{G})=n^{2}-1$. For $S U(2)$ there are three generators $T_{\mathfrak{a}}$, so explicitely we have

$$
\begin{aligned}
\operatorname{tr}\left(F_{a c}^{\mathfrak{a}} T_{\mathfrak{a}} U^{\mathfrak{b}} T_{\mathfrak{b}}\right) \operatorname{tr}\left(F^{c}{ }_{b}^{\mathfrak{a}} T_{\mathfrak{a}} U^{\mathfrak{b}} T_{\mathfrak{b}}\right) \\
\quad=K^{2} F_{a c}^{\mathfrak{a}} F^{c}{ }_{b}^{\mathfrak{c}} U^{\mathfrak{b}} U^{\mathfrak{d}} \delta_{\mathfrak{a} \mathfrak{b}} \delta_{\mathfrak{c} \mathfrak{d}}
\end{aligned}
$$

If the introduced auxiliary field $U^{\mathfrak{a}}$ satisfies the following algebra

$$
U^{\mathfrak{a}} U^{\mathfrak{b}}=\frac{1}{K} \delta^{\mathfrak{a} \mathfrak{b}},
$$

then Eq. (37) becomes

$$
\begin{aligned}
K^{2} F_{a c}^{\mathfrak{a}} F^{c} b^{\mathfrak{c}} & \frac{1}{K} \delta^{\mathfrak{b} \mathfrak{d}} \delta_{\mathfrak{a} \mathfrak{b}} \delta_{\mathfrak{c} \mathfrak{d}} \\
& =K F_{a c}^{\mathfrak{a}} F^{c}{ }_{b}^{\mathfrak{c}} \delta_{\mathfrak{a} \mathfrak{c}}=\operatorname{tr}\left(F_{a c}^{\mathfrak{a}} T_{\mathfrak{a}} F^{c}{ }^{\mathfrak{b}} T_{\mathfrak{b}}\right) .
\end{aligned}
$$

Hence, for the $S U(2)$ case the energy-momentum tensor (36) takes the standard form

$$
T_{a b}=\operatorname{tr}\left(F_{a c}^{\mathfrak{a}} T_{\mathfrak{a}} F_{b}^{c}{ }^{\mathfrak{b}} T_{\mathfrak{b}}+\frac{1}{4} \eta_{a b} F_{c d}^{\mathfrak{a}} T_{\mathfrak{a}} F^{c d \mathfrak{b}} T_{\mathfrak{b}}\right) .
$$

The algebra (38) of the auxiliary field $U^{\mathfrak{a}}$ has a simple geometrical interpretation. Let $u^{\mathfrak{a}}$ be orthonormal basis of the vector space $\mathbb{R}^{3}$, then the three vectors $U^{\mathfrak{a}}=u^{\mathfrak{a}} / \sqrt{K}$ automatically satisfy the relations (38). This can easily be generalized to $S U(n)$ by letting $u^{\mathfrak{a}}$ be the orthonormal vectors spanning the vector space $\mathbb{R}^{\operatorname{dim}(\mathscr{G})}$ of the same dimension as the Lie algebra of $\mathscr{G}$. Choosing the auxiliary field $U^{\mathfrak{a}}=u^{\mathfrak{a}} / \sqrt{K}$ allows us to construct all $S U(n)$ Einstein-Yang-Mills field equations consistently.

\section{REMARKS AND CONCLUSIONS}

Firstly, it should be noted that one could have tried to extend the Kaluza-Klein scheme in a different manner, namely to allow $\operatorname{dim}(\mathscr{G})$ extra dimensions rather than one extra dimension. However, such an approach does not yield the correct field equations by means of the contracted Bianchi identities, both in the torsion-free and the torsion case. Hence our auxiliary field approach is, as far as we know, the only one giving correct results without the need to introduce more than one additional spatial dimension.

The theory presented here is related to attempts to unify the four fundamental forces in a geometrical manner. However, for such an approach to work, requires the use of a gauge group that contains $U(1), S U(2)$ and $S U(3)$ as subgroups, such as the Georgi-Glashow model that is based on $S U(5)$. This model predicts a too fast proton decay rate and is therefore ruled out by experimental data. It, however, inspired other kinds of grand unification theories based on a variety of gauge groups, like $S O(10)$ (double covering of $\operatorname{Spin}(10)$ ) to mention the most prominent example, or the string inspired and more complicated $E_{6}$ that also contains $S O(10)$. From that point of view, our model can be seen as a geometrical argument in favor of Grand Unified Theories.

Before summarizing our result, we would like to emphasize that we followed two guiding principles: simplicity and agreement with experiment. The first has been taken into account when we neglected the $z$-component of the connection 1-form, while agreement with experiment has led to the choice of vanishing 4-dimensional torsion.

We showed that it is possible to extend the original Kaluza-Klein scheme to Yang-Mills theories. By considering a spacetime where the fifth dimension was allowed to carry torsion, we recovered the 4d Einstein-Maxwell equations from the contracted Bianchi identities. We introduced a set of auxiliary fields which enabled us to obtain the Einstein-Yang-Mills field equations, also by considering the contracted Bianchi identities. The dynamics of the introduced auxiliary fields still need to be studied in detail. From a geometrical point of view this is a particularly interesting aspect of the approach since it does not require the prescription of an underlying action principle. It is geometry that dictates the form of the field equations, contrary (but not in contradiction) to standard quantum field theories [26]. The key idea of this work, namely to introduce auxiliary fields rather than more and more additional dimensions to fit in the gauge fields, could be of special interest for a variety of today's models of theoretical physics.

\section{Acknowledgments}

We would like to thank Roy Maartens and Dmitri Vassiliev for valuable discussions. The work of CGB was supported by research grant BO 2530/1-1 of the German Research Foundation (DFG). 
[1] T. Kaluza, Sitzungsber. Preuss. Akad. Wiss. Berlin (Math. Phys. ) (1921) 966.

[2] O. Klein, Z. Phys. 37 (1926) 895 [Surveys High Energ. Phys. 5 (1986) 241].

[3] H. C. Lee, "An Introduction To Kaluza-Klein Theories." Proceedings, Workshop, Chalk River, Canada, August 11-16, 1983, World Scientific, Singapore (1984).

[4] T. Appelquist, A. Chodos and P. G. O. Freund, "Modern Kaluza-Klein Theories," Addison-Wesley, (1987).

[5] L. Fabbri, Annales Fond. Broglie 29 (2004) 641.

[6] H. Weyl, Sitzungsber. Preuss. Akad. Wiss. Berlin (Math. Phys. ) (1918) 465.

[7] H. F. M. Goenner, Living Rev. Rel. 7 (2004) 2.

[8] E. Cartan, Ann. Éc. norm. 40 (1923) 325.

[9] F. W. Hehl, P. Von Der Heyde, G. D. Kerlick and J. M. Nester, Rev. Mod. Phys. 48, 393 (1976).

[10] V. de Sabbata and C. Sivaram, "Spin and torsion in gravitation," World Scientific, Singapore (1994).

[11] D. Vassiliev, Phys. Rev. D 75 (2007) 025006.

[12] D. Vassiliev, Int. J. Geom. Meth. Mod. Phys. 4 (2007) 325.

[13] M. W. Kalinowski, Int. J. Theor. Phys. 20 (1981) 563.

[14] G. German, Class. Quant. Grav. 2 (1985) 455.

[15] C. Kohler, Int. J. Mod. Phys. A15 (2000) 1235.

[16] C. H. Oh and K. Singh, Class. Quant. Grav. 6 (1989)
1053.

[17] E. Schmutzer Exp. Tech. Phys. 30 (1982) 229.

[18] E. Schmutzer, in "Unified Field Theories of More than Four Dimensions", edited by E. Schmutzer and V. De Sabbata, World Scientific, Singapore (1983).

[19] V. De Sabbata and M. Gasperini, Phys. Rev. D 29 (1984) 171.

[20] D. Grumiller, W. Kummer and D. V. Vassilevich, Phys. Rept. 369 (2002) 327.

[21] H. Balasin, C. G. Böhmer and D. Grumiller, Gen. Rel. Grav. 37 (2005) 1435.

[22] F. W. Hehl, "A remark on an ansatz by M. W. Evans and the so-called Einstein-Cartan-Evans unified field theory," arXiv:physics/0612026.

[23] F. W. Hehl, "An assessment of Evans' unified field theory I," arXiv:physics/0703116.

[24] F. W. Hehl and Y. N. Obukhov "An assessment of Evans' unified field theory II," arXiv:physics/0703116.

[25] L. Fabbri, "On a completely antisymmetric Cartan tensor," arXiv:gr-qc/0608090.

[26] S. Weinberg, "The Quantum Theory of Fields. Vol. 1: Foundations," Cambridge University Press, Cambridge (1995). 